\title{
Evaluación diagnóstica de dos proteínas purificadas de Fasciola hepatica mediante ELISA en la fasciolosis ovina
}

\author{
FERNANDO FREDES*, JUAN ALARCÓN*, PAULA ILABACA* y HÉCTOR ALCAÍNO*
}

\section{DIAGNOSTIC EVALUATION OF TWO PURIFIED PROTEINS OF Fasciola hepatica BY MEANS OF ELISA IN THE SHEEP FASCIOLOSIS}

Two polipeptides (14 kDa and $29 \mathrm{kDa}$ ) of adult Fasciola hepatica were purified by electroelution and its diagnostics application through ELISA was evaluated. The sensibility was $60 \%$ and $94 \%$, and the specificity was $100 \%$ and $98 \%$, respectively. The $29 \mathrm{kD}$ a polypeptide was efficient for the diagnosis of the sheep fasciolosis, athough the $14 \mathrm{kDa}$ polypeptide was not, when using an ELISA test with it.

Key words: Immunodiagnosis, ELISA, electroelution, Fasciola hepatica, Antigens.

\section{INTRODUCCIÓN}

En Chile, la principal enfermedad parasitaria que afecta a las especies de abasto es la fasciolosis, causada por el trematodo Fasciola hepatica. $\mathrm{Su}$ relevancia radica además, en ser una enfermedad zoonótica ${ }^{1,2}$.

El diagnóstico de rutina de la fasciolosis se realiza mediante un examen coprológico de sedimentación, el cual es de un bajo costo y se basa en la detección de huevos eliminados por los parásitos adultos. Este método no tiene una sensibilidad adecuada, ya que no detecta los parásitos inmaduros que no producen huevos. Debido a esto en los últimos años se han buscado alternativas diagnósticas más eficientes y aplicables a gran escala. Es así como el uso de técnicas inmunológicas, permite el diagnóstico más temprano de esta parasitosis a nivel de masa, al detectar estados juveniles del parásito y con ello realizar la aplicación de tratamientos en forma más temprana y oportuna ${ }^{3-7}$.
Así por ejemplo, estas metodologías permiten diagnosticar la etapa prepatente y patente de la infección en los animales y en el hombre. Dentro de ellas las más eficientes y aplicables a gran escala han sido las inmunoenzimáticas ${ }^{3,4,6}$. Sin embargo, cuando se han empleado extractos antigénicos totales de $F$. hepatica, los resultados no han sido buenos. Lo anterior se debe a la aparición de reacciones con otros parásitos y también reacciones inespecífica, las que afectan la especificidad y sensibilidad, respectivamente de estas pruebas ${ }^{3,4,6}$. A través de los años se ha llegado a concluir, que la eficiencia de los métodos inmunológicos se ve afectada por la calidad del antígeno utilizado. Es decir, al ser este de mayor pureza se obtendrán mejores resultados ${ }^{8,9}$.

Mediante la electroelución en nuestro laboratorio logramos purificar estas dos proteínas, de 14 y de $29 \mathrm{kDa}$, y al evaluarlas mediante WB en la fasciolosis ovina obtuvimos una sensibilidad de $95 \%$ y $97,5 \%$ respectivamente y una especificidad del $100 \%$. Sin embargo, estas

* Departamento de Medicina Preventiva Animal, Facultad de Ciencias Veterinarias y Pecuarias, Universidad de Chile, Casilla 2, Correo 15, Santiago, Chile. E-mail: ffredes@uchile.cl

Financiado: Proyecto DID SAL 01/12-2 
proteínas aún no habían sido utilizadas en una prueba inmunodiagnóstica masiva, como es el ELISA $^{10}$. Por esto, en este trabajo se evaluó la eficiencia diagnóstica de ambas porteínas en un "test" de ELISA, utilizando sueros ovinos con fasciolosis, sin fasciolosis y sueros negativos a fasciolosis pero con otras parasitosis.

\section{MATERIAL Y MÉTODOS}

Preparación de antígenos E-S: En un matadero de la Región Metropolitana se recolectaron fasciolas vivas adultas. A partir de ellas, se obtuvieron los productos de excreciónsecreción (E-S). Para esto, las fasciolas fueron lavadas con suero fisiológico estéril y luego se incubaron por $16-18 \mathrm{hrs}$ a $37^{\circ} \mathrm{C}$, en solución de Hedon-Fleig estéril ${ }^{11}$. El sobrenadante obtenido, se dializó toda la noche a $4^{\circ} \mathrm{C}$ en agitación contra agua desionizada, y luego fue sometido a ultracentrifugación (15.000 G por $20 \mathrm{~min}$ ). A continuación se liofilizó, hasta obtener un volumen de $1 \mathrm{ml}$. Finalmente, se midió la concentración proteica del sobrenadante, para luego almacenarlo en alicuotas a $-70^{\circ} \mathrm{C}^{12}$.

SDS-PAGE: Una vez obtenidos estos productos (E-S), se realizó la técnica de $S D S$ $P A G E^{13}$. Fue llevada a cabo en mini geles de $8 \times 7 \times 1,5 \mathrm{~mm}$ con una concentración de poliacrilamida al $12 \%$. Los geles se colocaron en una cámara vertical Mini- Protean II (BIORAD), en condiciones denaturantes, con una peineta continua y a una diferencia de potencial de $100 \mathrm{~V}$ y un límite de $0,95 \AA$. La peineta que se ocupó tiene un carril central pequeño y dos laterales grandes. En los carriles laterales se colocó $150 \mu \mathrm{g}$ del preparado (E-S). En el carril central se incluyó una solución de marcador de peso molecular de origen comercial, cuyo rango va de 14,2 a $66 \mathrm{kDa}$. El gel fue cortado en tres trozos, dejando en el trozo del medio, el carril central y algo de los laterales. Luego el trozo central se tiñó con azul de Coomassie, para así poder determinar en que posición exacta se encuentran las proteínas de 14 y 29 kDa. Los otros dos trozos laterales sin teñir se cortaron al mismo nivel de las proteínas teñidas en cuadritos de $1 \mathrm{~cm}^{2}$ para ser sometidos a electroelución lo cual nos permite liberar las proteínas incluidas en los trocitos de gel.

Electroelución: Se realizó en un módulo de electroelución 422 (BIO-RAD) a 8-10 mA/tubo por 4 horas. Una vez obtenido el eluido se midió su concentración proteica ${ }^{12}$. Luego se realizó nuevamente un $S D S-P A G E$ para comprobar la eficiencia de la purificación ${ }^{13}$.

Finalmente, se agruparon los eluídos de cada proteína (14 ó $29 \mathrm{kDa}$ ) y se almacenaron a $-70^{\circ} \mathrm{C}$.

Sueros: 35 sueros de animales positivos a fasciolosis fueron determinados mediante inspección macroscópica post mortem de los hígados de ovinos faenados. Treinta y cinco sueros negativos a fasciolosis fueron obtenidos en la XII Región del país, zona libre de infección. Además se contó con 4 sueros de animales con hidatidosis, 4 con Thysanosoma actinioides y 4 con Cysticercus tenuicollis, todos ellos procedentes de la XII Región de Chile.

ELISA: Se empleó una técnica de ELISA en microplacas con 96 pocillos de fondo plano (12 $\mathrm{x}$ 8). Las placas se incubaron con $100 \mu \mathrm{ldel}$ antígeno semipurificado en cada pocillo. Este antígeno se diluyó en "buffer" carbonato a pH $9,6^{9}$. Las placas con el antígeno fueron incubadas por 3 horas a $37^{\circ} \mathrm{C}$. Luego se dejaron toda una noche a $4^{\circ} \mathrm{C}$, con agitación constante. Al día siguiente fueron lavadas con PBS-T20 al 0,05\% tres veces, 5 minutos cada vez. Luego fueron bloqueados los sitios activos, una hora a $4^{\circ} \mathrm{C}$, con una solución de PBS-T20 0,05\% más leche descremada al $3 \%$ en cámara húmeda. Posteriormente fueron lavadas 3 veces, con PBS-T20 al $0,05 \%$, se secaron, sellaron y fueron guardadas a $-20^{\circ} \mathrm{C}$.

En un primer ensayo se determinó la dilución óptima del antígeno, de los sueros y del conjugado una $\operatorname{IgG}$ anti ovina marcada con peroxidasa (SIGMA, MO, USA).

En la titulación para la proteína de $29 \mathrm{kDa}$ de la prueba de ELISA, se seleccionó en un primer momento $4 \mu \mathrm{g} / \mathrm{ml}$ de proteína, diluciones de $1 /$ 64 de suero y de 1/4.000 de conjugado. En el caso de la proteína de $14 \mathrm{kDa}$, la titulación dio 1 $\mu \mathrm{g} / \mathrm{ml}$ de proteína y dos diluciones de suero (1/ 32 y 1/64), pero una sola dilución de conjugado (1/4.000). El revelado se hizo con una solución de ortofenildiamina más $\mathrm{H}_{2} \mathrm{O}_{2}$, la reacción se detuvo al adicionar $25 \mu \mathrm{l}$ de ácido sulfúrico 2,5 M por pocillo y se llevó a un lector automático de ELISA a 492 nm, que nos entregó la densidad óptica ${ }^{9,14}$.

Los controles positivos utilizados fueron ovinos infectados experimentalmente que luego fueron sacrificados comprobando su positividad 
al examen posmortem. Los controles negativos fueron sueros de ovinos de la XII región.

El criterio que se empleó para determinar el punto de corte o "cut- off", fue considerar como positivo al suero que presentó una densidad óptica (DO) superior o igual a dos veces al promedio de la lectura de los sueros controles negativos. De esta forma, los sueros que arrojaron una DO bajo ese valor densidad fueron considerados negativos.

Analisis de resultados: Los resultados obtenidos por ELISA fueron comparados con el examen post morten de los hígados ovinos, mediante la prueba de Mc Nemar para proporciones correlacionadas o dependientes ${ }^{15}$. Con los datos obtenidos se calculó la sensibilidad, especificidad y valores predictivos positivos y negativos de las proteínas seleccionadas ${ }^{16}$.

\section{RESULTADOS}

Con la proteína de $29 \mathrm{kDa}$ la prueba de Mc Nemar permitió establecer que no existen diferencias diagnósticas significativas entre el examen post mortem y el ELISA realizado ( $\mathrm{p}>$ 0,05). Así también con la proteína de $29 \mathrm{kDa}$ se obtuvo una sensibilidad de $94 \%$, una especificidad del $98 \%$ y valores predictivos positivos de $97 \%$ y negativos de $96 \%$.

En el caso de la proteína de $14 \mathrm{kDa}$, mediante Mc Nemar indicó que existía diferencia significativa entre el ELISA y el examen post mortem $(\mathrm{p}<0,05)$. Además la proteína de 14 $\mathrm{kDa}$ dio valores de sensibilidad y de especificidad de un $60 \%$ y un $100 \%$ respectivamente. El valor predictivo positivo fue de un $100 \%$ y el negativo de $77 \%$.

\section{DISCUSIÓN}

Según diversos trabajos realizados por distintos autores es posible señalar, que la detección de anticuerpos en el suero es el método de elección para el diagnóstico de $F$. hepatica ${ }^{3-10,14,17}$. En este trabajo se combinó el método de purificación por electroelución con una prueba de ELISA, para así con sueros detectores evaluar nuestros antígenos por sus resultados de inmunoreacción en una prueba de ELISA.

$\mathrm{Al}$ igual que en los estudios anteriores, se ha evidenciado que se obtienen una serie de proteínas a partir del antígeno completo de E-S ${ }^{18}$. Nuestro estudio se centró en la purificación de las proteínas de 14 y $29 \mathrm{kDa}$, porque estas se han destacado por su eficiencia diagnóstica y su alta concentración $^{9,10}$. Sin embargo, en nuestro país no habían sido evaluadas mediante ELISA, separadamente en el inmunodiagnóstico de la fasciolosis animal, salvo mediante WB en la que se obtuvieron excelentes resultados ${ }^{10}$.

Utilizando la electroelución, es posible obtener cantidad de antígeno suficiente para realizar la sensibilización de las placas del ELISA. Se debe considerar además el tiempo de corrida del gel (SDS-PAGE) que permitirá obtener los trozos de gel necesarios para realizar la electroelución.

En estudios realizados anteriormente en el laboratorio de parasitología de nuestra Facultad, se han usado estas proteínas de 14 y $29 \mathrm{kDa}$, semipurificadas por cromatografía de exclusión, pero ambas incluidas en un mismo eluído ${ }^{9,14}$. Estas proteínas presentaron altos valores de sensibilidad y especificidad mediante ELISA $(94,5 \%$ y $93,5 \%$ respectivamente $)^{9}$. Además de ser reconocidas, mediante WB, desde la 6 semana de infección para la proteína de $29 \mathrm{kDa}$ y a partir de los 2 meses en el caso de la proteína de $14 \mathrm{kDa}^{14}$.

Las proteínas utilizadas en nuestros estudios, fueron definidas anteriormente como especificas en ovinos, equinos y cerdos ${ }^{14,19}$.

Los resultados obtenidos son similares en sensibilidad y especificidad con la proteína de 29 $\mathrm{kDa}$ a los obtenidos mediante WB que fueron de $97,5 \%$ y $100 \%$ respectivamente ${ }^{10}$. Mientras que nuestros valores para esta proteína son de $94 \%$ y $98 \%$, lo cual puede explicarse por que la técnica de ELISA es menos sensible que WB.

La sensibilidad para la proteína de $14 \mathrm{kDa}$ fue más baja en este estudio (60\%), que la obtenida mediante WB que fue de un $95 \%$ de sensibilidad ${ }^{10}$. La especificidad en nuestro trabajo y con WB fue de un $100 \%{ }^{10}$.

Sería importante en estudios futuros determinar las posibles causas de la baja en la sensibilidad de la proteína de $14 \mathrm{kDa}$ respecto a lo obtenido por WB anteriormente en nuestro laboratorio ${ }^{10}$, ya que estos valores no permitirían su uso en el inmunodiagnóstico por ELISA, lo que la descarta de su empleo para el diagnóstico masivo.

En tanto los altos valores de sensibilidad y especificidad mediante ELISA de la proteína de $29 \mathrm{kDa}$, hace que esta proteína pueda ser utilizada en el diagnostico de $F$. hepatica mediante esta prueba. Por lo que en estudios posteriores se podrían realizar diagnósticos masivos con "kits" 
de ELISA elaborados con esta proteína.

\section{RESUMEN}

Dos proteínas antigénicas de Fasciola hepatica, una de $14 \mathrm{kDa}$ y otra de $29 \mathrm{kDa}$, fueron purificadas por la técnica de electroelución a partir de un antígeno de excreción- secreción (ES) de fasciolas adultas. Estas proteínas fueron evaluadas por separado, mediante una técnica de ELISA utilizando para ello sueros de ovinos con fasciolosis, sueros de ovinos sin fasciolosis y sueros de ovinos con otras parasitosis. La proteína de $14 \mathrm{kDa}$ tuvo una sensibilidad baja de un $60 \%$ y una especificidad del $100 \%$, mientras que su valor predictivo positivo fue de un $100 \%$ y el negativo de $77 \%$. Los valores de sensibilidad y especificidad obtenidos para la proteína de 29 $\mathrm{kDa}$ fueron de un $94 \%$ y $98 \%$ respectivamente, mientras que el valor predictivo positivo fue de un $97 \%$ y el negativo de un $96 \%$. Finalmente, se pudo concluir que la proteína de $14 \mathrm{kDa}$ no mostró ser útil en el inmunodiagnóstico de esta enfermedad mediante ELISA. En cambio la proteína de $29 \mathrm{kDa}$, debido a sus altos valores de sensibilidad y especificidad, puede ser utilizada en el inmunodiagnóstico de la fasciolosis animal mediante la técnica de ELISA.

\section{REFERENCIAS}

1.- MORALES M A, LUENGO J. Beneficio y causales de decomisos de ovinos, porcinos, equinos, caprinos y camélidos en Chile. Av. Cs Vet 1995; 11: 136-46.

2.- ALCAÍNO H, APT W. Algunos antecedentes sobre la fasciolosis animal y humana. Monog Med Vet 1989; 11: $14-29$.

3.- GORMAN T, WENZEL J, LORCA M et al. Pruebas de inmunoprecipitación y hemoaglutinación indirecta en el diagnóstico de la fasciolosis ovina. Parasitol al Día 1990; 14: 51-6.

4.- GORMAN T, BRAVO J, LORCA M et al. Diagnóstico de la fasciolasis de equinos y porcinos mediante dobledifusión, contrainmunoelectroforesis y hemoaglutinación indirecta. Arch Med Vet 1991; 23: 123-30.

5.- RUIZ-NAVARRETE M, ARRIAGA C, BAUTISTA C.; MORILLA A. Fasciola hepatica: Characterization of somatic and excretory-secretory antigens of adult flukes recognized by infected sheep. Rev Lat Amer Microbiol 1993; 35: 301-7.
6.- GORMAN T, MORENO P, LORCA M et al. Inmunodiagnóstico de la fasciolosis animal mediante una prueba inmunoenzimática (ELISA). Parasitol al Día 1991; 15: 87-93.

7.- BAUTISTA-GARFIAS C, LÓPEZ-ARELLANO M SÁNCHEZ-ALBARRÁN A. A new method for serodiagnosis of sheep Fasciolasis using helminth excretory-secretory products. Parasitol Res 1989; 76: 135-7.

8.- GORMAN T, JAQUE S, FREDES F, SILVA M. Importancia diagnóstica de fracciones antigénicas de Fasciola hepatica separadas por cromatografía de exclusión por tamaño molecular y analizadas mediante inmunoelectrotransferencia en condiciones nativas y de denaturación-reducción. Parasitol al Día 1996; 20: 38-44.

9.- FREDES F, GORMAN T, SILVA M, ALCAÍNO H. Evaluación diagnóstica de fracciones cromatográficas de Fasciola hepatica mediante Western Blot y ELISA en animales infectados. Arch Med Vet 1997; 29: 283-94.

10.- FREDES F, SÁNCHEZ C, GORMAN T, ALCAÍNO H. Purificación de antígenos de Fasciola hepatica mediante electroelución y su aplicación inmunodiagnóstica en la infección animal. Parasitol al Día 2001; 25: 19-23.

11.- MORILLA A, BAUTISTA C. Manual de Inmunología. Ed. Diana, México. 1986. 397 pp.

12.- BRADFORD M. A rapid and sensitive method for quantitation of microgram quantities of protein utilizing the principle of protein dye-binding. Anal Biochem 1976: 72: 248-54.

13.- TSANG V, HANCOCK K, WILSON M et al. In: Immunology series $\mathrm{N}^{\circ} 15$. Procedural Guide. U.S. Department of Health and Human Services, Atlanta, Georgia. 1986. 25 p.

14.- GORMAN T, LÓPEZ C, FREDES F, ALCAÍNO H. Monitoreo inmunológico del éxito terapéutico en fasciolosis ovina empleando un antígeno semipurificado de < 30 kda. Parasitol al Día 2000; 24: 27-34.

15.- REMINGTON R D, SHORK MA. Chi-square test for frecuency data. In: Statistics with applications to the biological and health sciencies. Englewood Cliffs, N. J. Prentice-hall. 1970. pp: 240-4.

16.- THRUSFIELD M. Epidemiología Veterinaria. Ed. Acribia, S.A. Zaragoza, España. 1990. 339 p.

17.- QURESHI T, WAGNER G, LYNN DRAWE D et al. Enzyme-linked immunoelectrotransfer blot analysis of excretory-secretory proteins of Fascioloides magna and Fasciola hepatica. Vet Parasitol 1995; 58: 357-63.

18.- GORMAN T, CONCHA V, FREDES F et al. Detección de antígenos de interés diagnóstico en infecciones animales por Fasciola hepatica. Parasitol al Día 1994; 18: $26-32$.

19.- SANTIAGO N, HILLYER G. Isolation of potential serodiagnostic Fasciola hepatica antigens by electroelution from polyacrylamide gels. Am J Trop Med Hyg 1986; 35: 1210-7. 